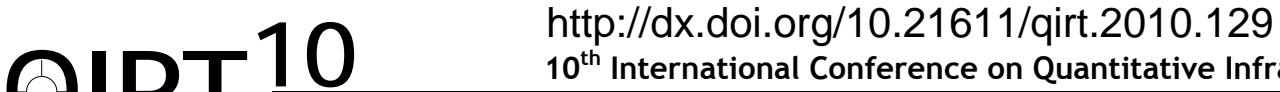 \\ $10^{\text {th }}$ International Conference on Quantitative InfraRed Thermography \\ July 27-30, 2010, Québec (Canada)
}

\section{Cross-correlation based compression technique for frequency modulated thermal wave imaging}

\author{
by Ghali Venkata Subbarao*, Ravibabu Mulaveesla* and Masahiro Takei**
}

*Electronics and Communication Engineering Research Group., PDPM-Indian Institute of Information Technology Design and Manufacturing, Jabalpur., Dumna, Khamaria p.o., India-482005, *ravibabucareiitd@yahoo.co.in

${ }^{\star *}$ Department of Mechanical Engineering, College of Science and Technology, Nihon University, 1-8-14, Kanda Surugadai, Chiyoda-ku, Tokyo, 101-8308, Japan, **masa@mech.cst.nihon-u.ac.jp

\begin{abstract}
Infrared non destructive testing (NDT) with recently proposed frequency modulated thermal wave imaging, launches thermal waves with significant energy in the desired band of frequencies, decided by sample thermal characteristics and its dimensions, in the object under test. Pulse compression based post processing enhances the defect detection capability of the above scheme even with moderate peak power excitation sources. This contribution highlights the applicability of the correlation based pulse compression scheme for investigation of defects like voids, inclusions and cracks and comparison has been made with existing phase based analysis.
\end{abstract}

Key words: Infrared thermography, Frequency modulated thermal wave imaging, Phase image, Pulse compression.

\section{Introduction}

Thermography has been successfully used as a non destructive testing method to evaluate the structural integrity and assessment of subsurface anomalies. Its reliance on thermal properties of substances facilitates its applicability to a number of solid materials. In active IR Thermography, subsurface abnormalities have been identified by processing the captured thermogram sequence generated by thermal mapping of the object under examination for the known input heat stimulus. Presently, three different approaches of active thermal nondestructive techniques predominant in use are: Pulse Thermography (PT), lock in Thermography (LT) and Pulse Phase Thermography (PPT) [1]. In pulse thermography [2], the tested material is warmed or cooled with a short duration energy pulse (example optical, induction heating and ultra sonic heating etc.) and a measurement of the temporal evolution of the surface temperature is performed with an infrared (IR) camera. The surface temperature gradients on the sample help to localize the defects inside the material. However the surface temperature gradients are caused not only because of hidden defects but also affected by local variations of emissivity on the material surface as well as due to non-uniform heating. Lock-in thermography uses periodic sinusoidal thermal excitation in order to derive information of reflected thermal wave phase and magnitude [3] even at considerably low peak powers. The phase angle has the advantage of being less sensitive to local variations of illumination or of surface emissivity. Because of its mono-frequency excitation, the depth resolution of the experiment in a run gets fixed (i.e. fixed 'thermal wavelength'). To detect defects located at various depths in the test sample, repetition of the test at various frequencies becomes a time consuming process. The experimental arrangement for pulse phase thermography [4] is similar to that of pulse thermography, but the fundamental idea of processing the captured image sequence during the experiment, is different. In PPT, the extraction of various frequency components in the captured sequence is performed by Fourier transform on each pixel of the thermal image sequence [5]. The phase images thus obtained show all the merits of the phase images obtained with LT (less sensitive to surface in-homogeneities and variations in illumination). Theoretically, the short duration excitation pulse in PPT does launch a large number of frequency components into the test samples, but the higher order components usually do not have sufficient energy to propagate deep into the sample. To overcome some of the traditional limitations of conventional thermal wave imaging techniques (resolution, peak power, depth of penetration), the present work focuses on non-stationary forms of thermal excitation technique: frequency modulated thermal wave imaging (FMTWI) [6-8].

This paper presents simulated results obtained for the recently introduced three dimensional pulse compression for detection of artificial defects incorporated in plain carbon steel and compared them with phase based approach.

\section{Frequency modulated thermal wave imaging}

Active infrared non-destructive testing involves submission of the object to known thermal excitation and thermal mapping of the superficial temperature with an infrared camera. Requirement of larger powers for deeper depth probing, and limitations with high peak power sources promoted probing of low frequency, small average powers for longer duration. Excitation schemes which can probe lower peak powers and gathers incident energies into a pseudo pulse are better 
alternatives for high peak power requirements. FMTWI introduces thermal waves of a suitable band of frequencies of equal energy to probes into the sample for detection of subsurface abnormalities at different depths in a single experimentation cycle. Pulse compression for FMTWI [7] concentrates the energy of the signal about a time instant, produces a pseudo pulsed response from this long duration excitation and also enhances signal to noise ratio (SNR).

\subsection{Frequency modulated thermal wave}

In FMTWI, frequency modulated heat flux deposited over the sample generates thermal waves as a result of thermal oscillations at surface. These generated thermal waves diffuse to interior portions of the substances under test in addition it produces similar time varying temperature distribution on the surface. Under a suitable defect and sample dimensions thermal diffusion in substances can be explained with one dimensional parabolic Fourier heat conduction equation given by

$$
\frac{\partial^{2} T(x, t)}{\partial x^{2}}=\frac{1}{\alpha} \frac{\partial T}{\partial t}
$$

Where $\alpha$ the thermal diffusion coefficient and $\mathrm{T}$ is is the instantaneous temperature on the surface and $x$ is direction of heat flow (perpendicular to the surface). On solving the above equation for FMTWI under stipulated boundary conditions results in

$$
T(x, t)=T_{0} e^{-x \sqrt{\frac{\pi}{\alpha}\left(f+\frac{B t}{\tau}\right)}} e^{-j x \sqrt{\frac{\pi}{\alpha}\left(f+\frac{B t}{\tau}\right)}} e^{2 \pi j\left(f t+\frac{B t^{2}}{2 \tau}\right)}
$$

Where $\mathrm{B} t$ is the frequency sweep rate of the chirp and $\tau$ is duration of excitation.

Penetrating thermal waves gets weakened due to larger attenuations from the substance. The depth at which energy of the wave attenuates to $1 / e$ times of its surface value is called thermal diffusion length, which plays a vital role in thermography. Thermal diffusion length of this frequency modulated thermal wave is given by

$$
\mu_{\mathrm{fm}}=\sqrt{\alpha / \pi(\mathrm{f}+\mathrm{B} \mathrm{t} / \tau)}
$$

Dependence of thermal diffusion length on frequency and band width of the applied signal facilitates the depth resolution of probing of different depths simultaneously, within a single sweep using FMTWI.

At the boundary of anomalies, the forward diffusive thermal front encounters reflection due to difference in thermal effusivity or thermal impedance mismatch between the materials. Resultant temperature response on the surface due to reflected and surface existing waves generates a delayed thermal wave depending on the depth of the defect, with respect to the incident wave form. The resultant temperature distribution over the sample results hot or cold spots at the positions of the anomalies, individuates them by a relative contrast. To avoid the effect of non uniform heating and to analyse contributions from the deeper defects, suitable post processing over captured data has to be performed.

\subsection{Pulse compression}

Pulse compression concentrates the total applied energy into a narrow time slot using matched filtering and improves depth resolution by a ratio of the half width of the sinc to applied duration of excitation. i.e $T^{\prime} / T$ where as $T^{\prime}$ is the half width of the sinc and $T$ is the duration of the applied chirp as shown in Fig 1. In this approach, a linear frequency modulated chirp is convolved with its flipped replica produces a pseudo pulse (i.e sinc) response centered about a group delayed time instant.
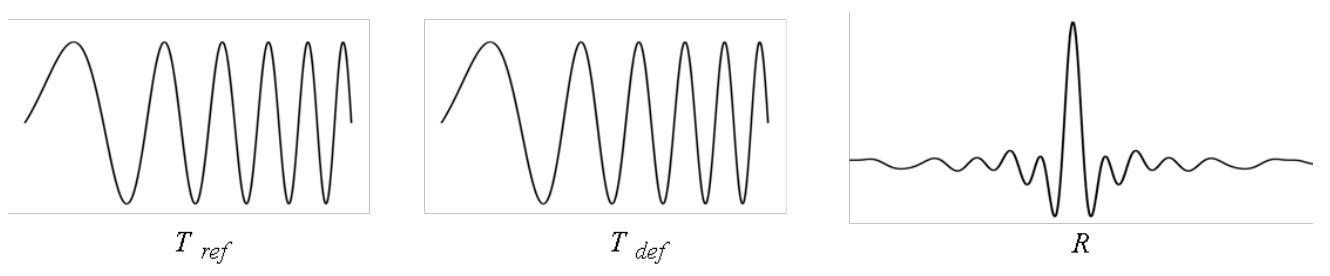

Fig. 1. Pulse compression through correlation using matched filtering 
Matched filter improves the SNR of the received signal by $T B$ and contributes for better detection of even deeper defects. In case of symmetric signals, similarity between the signals has been assessed using correlation. In three dimensional pulse compression approach, temporal thermal response corresponding to each pixel in view has been cross correlated with a reference pixel thermal profile. The time lag produced by the defective contributes for their normalized cross correlation coefficient variations depending on the depth of the defect. These variations individuate defects by producing different contrast over defect and sound portions in the images obtained from correlation process.

Consider $T_{\text {ref }}, T_{\text {def }}$ are the thermal profiles of non defective and defective pixels respectively. Propagation time of thermal wave for defect located at a particular depth introduces corresponding time lag with respective to reference thermal response can be assumed to be $T_{r e f}(t), T_{d e f}\left(t-t^{\prime}\right)$. Cross correlation of the sequences is obtained by

$$
\mathrm{R}(\mathrm{t})=\tau \int_{-\infty}^{\infty} \mathbb{T}_{\text {ref }}\left((\tau) \mathrm{t}_{\mathrm{def}} \mathrm{t}^{\prime}\right) d \tau-
$$

In order to exhibit the group delayed thermal response, cross correlated response of defective pixel has been compared with the auto correlation of the non defective pixel response. Auto correlation of non defective pixel response centered about zero clearly separated from the defective pixel cross correlation response has been used for depth quantification of the defects as it is unaffected by random noise, non uniform heating and surface emissivity variations over the sample [7]. This approach preserves the merits of phase approach in addition to the compression of the energy to localized time instant which enhances the relative contrast between defective and sound regions.

\section{Results and discussion}

In order to test the applicability of the correlation based pulse compression processing with frequency modulated thermal wave imaging for voids, cracks and inclusions etc., a plain carbon steel sample of thickness $2 \mathrm{~cm}$ has been modeled by incorporating a void, an inclusion and a crack as shown in figure 2(a). Finite element based thermal analysis has been performed on steel sample and heat flux of $2000 \mathrm{~W} / \mathrm{m}^{2}$ with a linear frequency variation from $0.01 \mathrm{~Hz}$ to $0.1 \mathrm{~Hz}$ in $100 \mathrm{~s}$ duration is imposed onto the modeled sample further image sequence has been captured at a frequency of $10 \mathrm{~Hz}$.

In pulse compression based analysis, cross correlation has been performed between the temporal thermal profiles of each pixel with a pre selected reference thermal profile (of a non defective pixel), after removing the dc component from each profile and a new processed image sequence is constructed. A correlation image, which better represents the defects, is identified for detection of subsurface anomalies. Correlation image represents the normalized correlation coefficients of the corresponding pixels at a group delayed instant. Phase analysis has been performed by the application of fast Fourier transform (FFT) on thermal profiles of each pixel in view. Figure 2(b) represents the correlation image at a group delayed instant of 2s for the steel sample shown in figure 2(a). It is clear that reflection from inclusion individuates its thermal property discrimination from that of the void. Where as the obtained phase image from the FFT analysis, on the captured temporal response is as shown in figure 2(c).

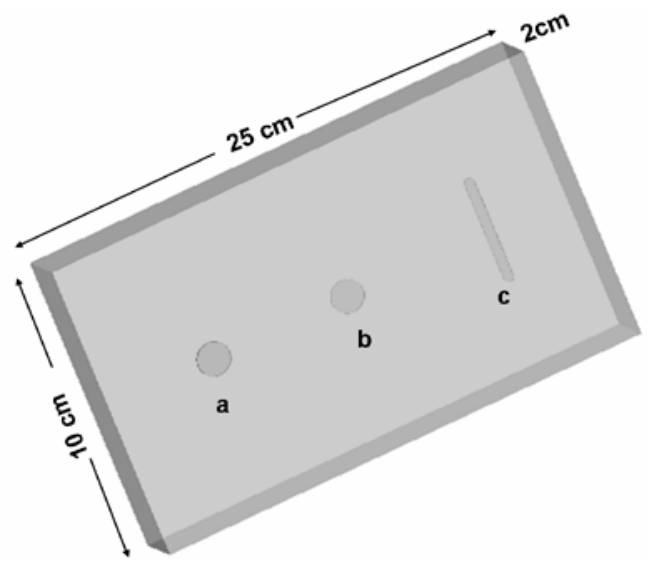

a 

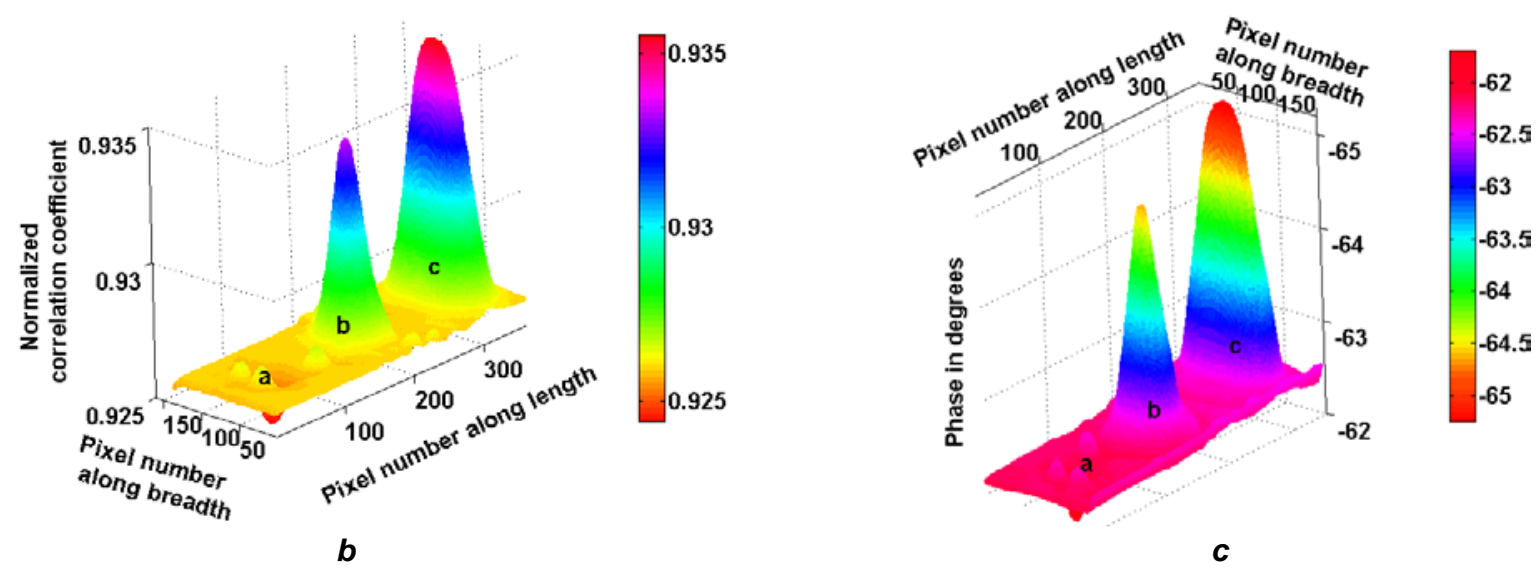

Fig. 2.(a) Layout of the simulated plain carbon steel sample with a. tungsten inclusion and b. void of diameter $1 \mathrm{~cm}$ and thickness $1 \mathrm{~mm}$ each and c. a crack of width $0.5 \mathrm{~cm}$ and length $5 \mathrm{~cm}$,all are at depth $7.5 \mathrm{~mm}$ 2.(b) Correlation image at a group delayed instant of 2s 2 .(c) Phase image obtained at frequency $0.06 \mathrm{~Hz}$.

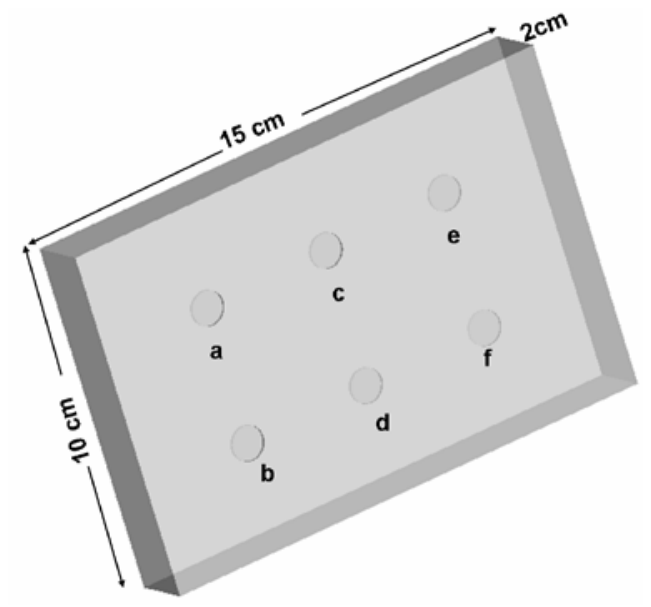

a

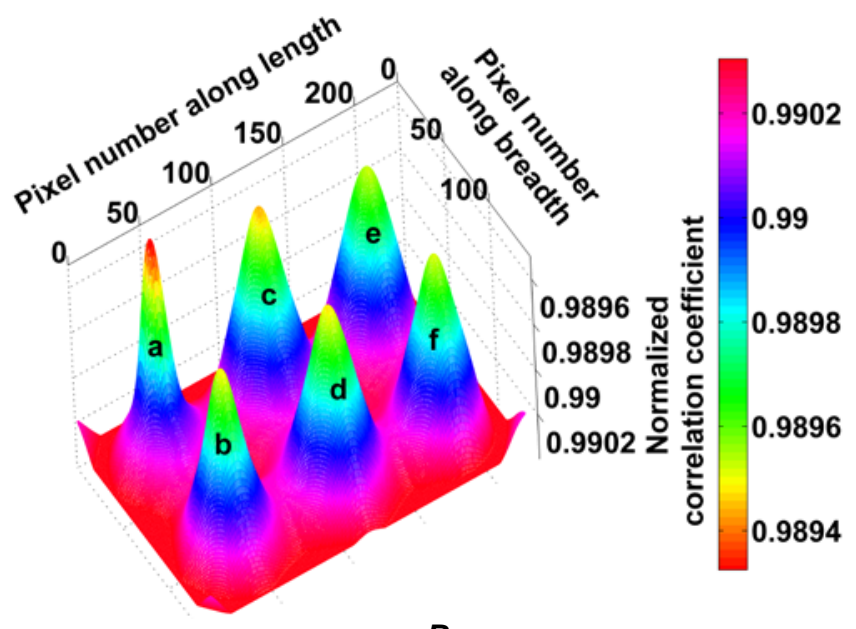

$B$

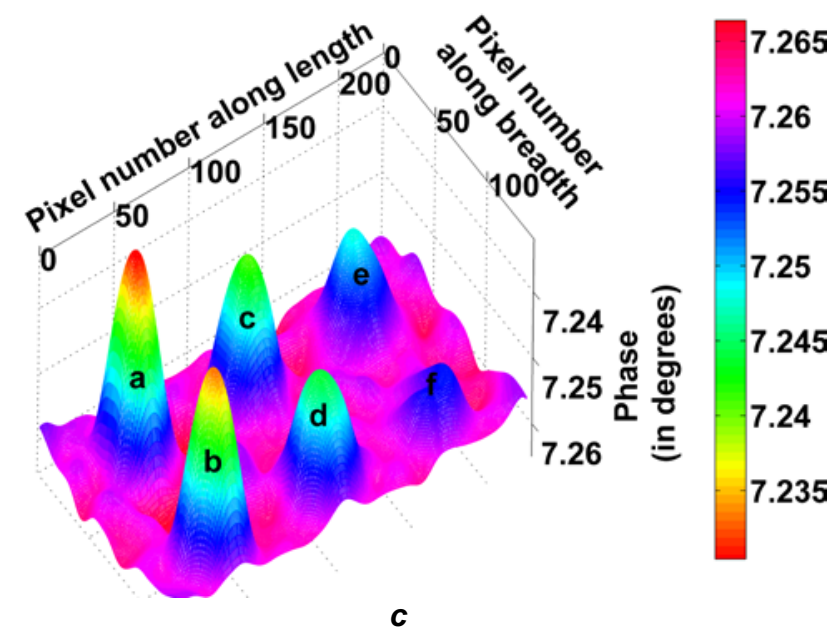

Fig. 3. (a) Layout of the simulated plain carbon steel sample with voids at depths a at $3 \mathrm{~mm}, b$ at $4 \mathrm{~mm}, \mathrm{c}$ at $5 \mathrm{~mm}$,d at $6 \mathrm{~mm}, \mathrm{e}$ at $7 \mathrm{~mm}$, f at $8 \mathrm{~mm}$. 3.(b) Correlation image at a group delayed instant of $0.2 \mathrm{~s}$ 3.(c) Phase image obtained at frequency $0.1 \mathrm{~Hz}$. 
Further a plain carbon steel sample of thickness $2 \mathrm{~cm}$ has been modeled with cylindrical voids of thickness $1 \mathrm{~mm}$ and diameter $1 \mathrm{~cm}$ with dimensions as shown in figure3(a). In order to investigate the capability of FMTWI using pulse compression processing, sample has been excited with a frequency modulated heat flux of amplitude $2000 \mathrm{~W} / \mathrm{m}^{2}$ with linear frequency variation from $0.01 \mathrm{~Hz}$ to $0.1 \mathrm{~Hz}$ in 100s duration. Larger normalized correlation coefficient resembles the depth of the defect from surface i.e larger the coefficient lesser is the depth from the surface. Broadening due to lateral diffusion influence from the deeper defects is clear from the defects in figure3 (b) and 3(c). More spreading is observed with deeper defects. Noise floor of phase image of figure 3(c) reduced the SNR of the defects compared to figure 3(b), as shown in Table (1).

Inclusions are modeled by filling air gaps of the previously modeled sample with tungsten as shown in figure 4(a) and thermal mapping of the surface has been performed by imposing a frequency modulated heat flux of amplitude 2000 $\mathrm{W} / \mathrm{m}^{2}$ with a linear frequency sweep form 0.01 to $0.1 \mathrm{~Hz}$ in $100 \mathrm{~s}$. Normalized correlation coefficients of inclusions in figure 4(b) are relatively lower than that of voids represents variation in thermal properties of the inclusion material, even though they are at same depths as shown in figure 3(a). It is observed from figures 4(b) and 4(c), that the lateral diffusion and noise floor in correlation image is less compared to that of the phase image.

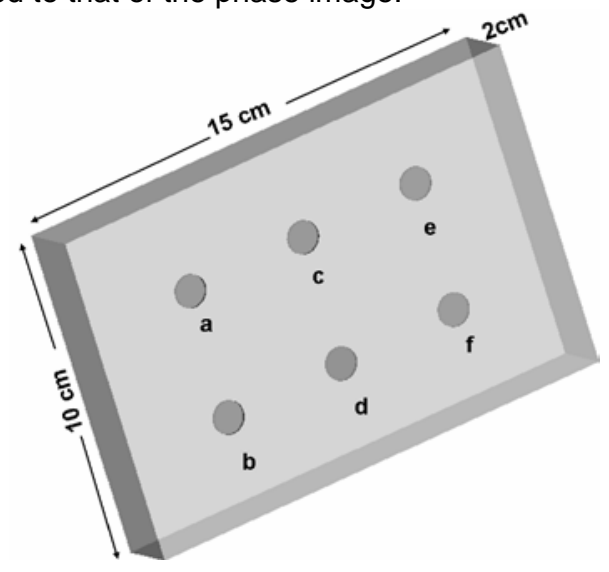

a

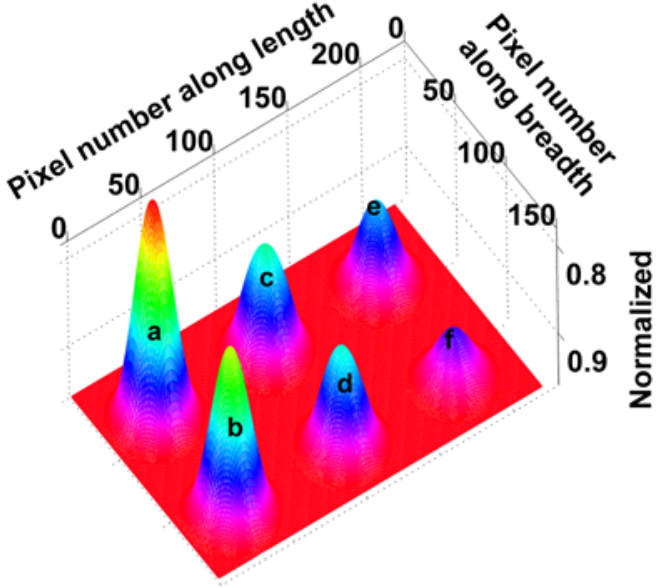

b

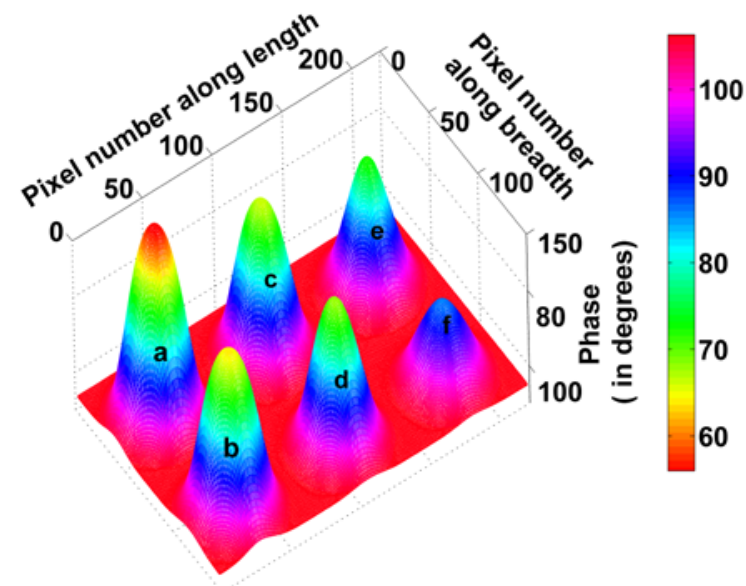

C

Fig. 4. (a) Layout of the simulated plain carbon steel sample with tungsten inclusions at depths a at $3 \mathrm{~mm}, b$ at $4 \mathrm{~mm}, c$ at $5 \mathrm{~mm}, \mathrm{~d}$ at $6 \mathrm{~mm}$, e at $7 \mathrm{~mm}$, f at $8 \mathrm{~mm}$ 4.(b) Correlation image at a group delayed instant of 0.6s 4.(c) Phase image obtained at frequency $0.05 \mathrm{~Hz}$. 
In order to verify the applicability of the technique to detect cracks, a plain carbon steel sample has been modeled with four cracks of length $3 \mathrm{~cm}$, width $0.3 \mathrm{~cm}$ and of thickness $1 \mathrm{~mm}$ kept at different depths from the top surface as shown in figure 5(a). It is clear from correlation image obtained with a group delay of $1 \mathrm{~s}$ for detection of signatures of crack defects is as shown in figure 5(b), It is clear that all the cracks are visible with enough contrast. Where as in phase image of figure 5(c), defects are contaminated in noise. SNR of defects in phase image are less than that of the correlation image as shown in table (1). Noise in temporal thermal profiles are reducing the ability of the detection has been clearly observed with phase images than correlation images as same thermal profiles have been used for processing in both approaches. Signal to noise ratio of the defects has been computed from

$$
\text { SNR }=\frac{\text { (Mean of the defective area }- \text { Mean of the non defective area) }}{\text { Standard deviation of non defective area }}
$$

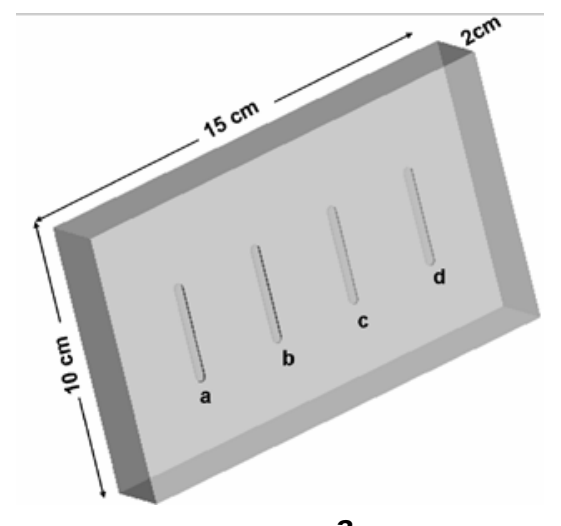

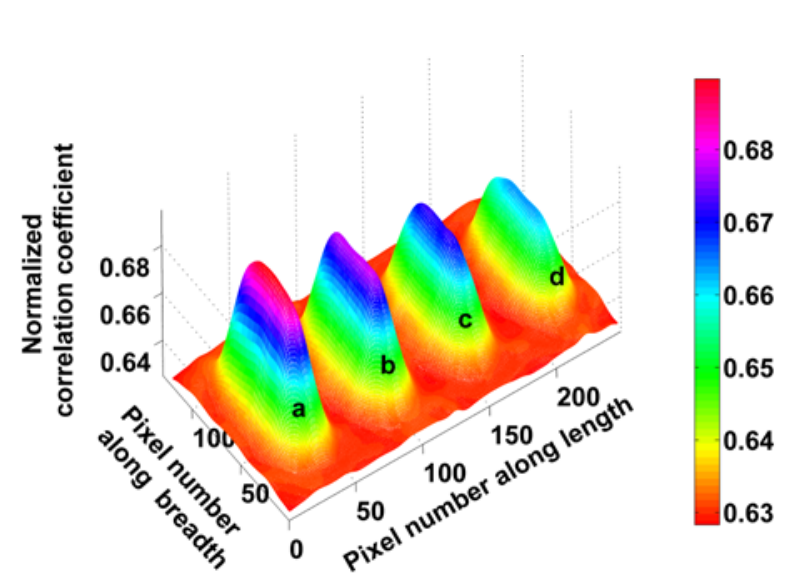

b

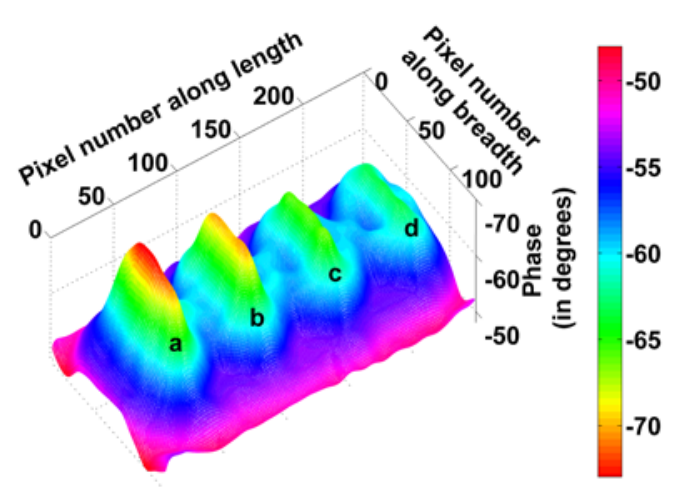

C

Fig. 5. (a) Layout of the simulated plain carbon steel sample with cracks at depths a at $3 \mathrm{~mm}, b$ at $4 \mathrm{~mm}, \mathrm{c}$ at $5 \mathrm{~mm}, d$ at $6 \mathrm{~mm}$. 5. (b) correlation image at a group delayed instant of 1s 5. (c) Phase image obtained at frequency $0.1 \mathrm{~Hz}$.

Comparison of SNR's in different cases is given in table (1). It is proved that the correlation based processing is superior to Phase based approach for FMTWI. 
Table 1. SNRs of defects

\begin{tabular}{|c|c|c|c|c|c|c|}
\hline \multirow{2}{*}{ Defect } & \multicolumn{6}{|c|}{ SNR of the defect $(d B)$} \\
\cline { 2 - 7 } & \multicolumn{2}{|c|}{ Voids } & \multicolumn{2}{c|}{ inclusions } & \multicolumn{2}{c|}{ cracks } \\
\cline { 2 - 7 } & Correlation & Phase & Correlation & Phase & Correlation & Phase \\
\hline$a$ & 47.16 & 53.34 & 39.77 & 29.78 & 57.49 & 38.43 \\
\hline$b$ & 49.89 & 48.42 & 34.98 & 27.58 & 55.63 & 38.21 \\
\hline$c$ & 49.30 & 34.48 & 31.31 & 36.7 & 51.36 & 35.13 \\
\hline$d$ & 48.72 & 35.59 & 26.47 & 30.61 & 51.17 & 33.66 \\
\hline$e$ & 48.52 & 30.6 & 24.31 & 27.02 & --- & --- \\
\hline$f$ & 45.89 & 20.76 & 18.37 & 22.05 & --- & -- \\
\hline
\end{tabular}

\section{REFERENCES}

[1] Maldague X.P.V., Theory and Practice of Infrared Thermography for Nondestructive Testing Wiley-Interscience, Hoboken, 2001.

[2] Pickering S, Almond D. P.," Matched excitation energy comparison of the pulse and lock-in thermography NDE techniques," NDT\&E International.Vol.79, pp.501-509, 2009.

[3] Busse G., Wu D. and Karpen W., "Thermal wave imaging with phase sensitive modulated thermography," J. Appl. Phys. Vol. 71, pp. 3962-3965, 1992.

[4] Maldague, X.P.V. and Marinetti, S., "Pulse phase thermography", J. Appl. Phys. 79 (5) , pp. 2694-2698, 1996.

[5] Ibarra-Castanedo C., Avdelidis N. P., Maldague X. P. V., "Qualitative and quantitative assessment of steel plates using pulsed phase thermography," J. Materials Evaluation, 63(11), pp. 1128-1133, 2005.

[6] Mulaveesala R. and Tuli S., "Theory of frequency modulated thermal wave imaging for non-destructive subsurface defect detection," Appl. Phys.Lett. 89, No.19, pp. 1913, 2006.

[7] Mulaveesala R., Vaddi J. S. and Singh P., "Pulse compression approach to infrared nondestructive characterization," Rev. Sci. Instrum., vol. 79, No. 09, pp. 4901, 2008.

[8] Ghali, V.S., Jonnalagadda., N. and Mulaveesala R., "Three-Dimensional Pulse Compression for Infrared Nondestructive Testing", IEEE Sensors Journal, vol.9(7) , pp.832-833, 2009. 